\title{
DISJOINT MEROMORPHIC FUNCTIONS AND NONOSCILLATORY DIFFERENTIAL SYSTEMS
}

BY

\section{AHARONOV AND M. LAVIE( ${ }^{*}$ )}

ABSTRACT. Conditions satisfied by disjoint meromorphic functions are obtained. These results are applied to nonoscillatory differential systems and disfocal differential equations.

1. Introduction. In the first part of this paper we are concerned with meromorphic functions which are disjoint in the unit disk. Making use of a recent result for pairs of functions [11], we obtain in $\$ \S 3$ and 4 necessary conditions satisfied by disjoint meromorphic functions.

Disjoint functions have been recently applied to oscillation problems of linear differential system and equations [6], [8]. Applying some of our function theoretic results, we obtain in the second part of our paper necessary conditions for a differential systems to be nonoscillatory in the unit disk.

Finally, in $\$ 5$, necessary conditions for a linear differential equation to be disfocal in the unit disk are given and some examples are considered.

2. Disjoint functions and pairs of functions. Let $f(z)$ and $g(z)$ be meromorphic functions in the unit disk $U$, such that

$$
f(z) \neq g(\zeta), \quad|z|,|\zeta|<1,
$$

then we say that $f(z)$ and $g(z)$ are disjoint meromorphic functions in $U$.

Since the Möbius transformation

$$
T w=(a w+b) /(c w+d), \quad a d-b c \neq 0
$$

is one to one in the whole complex plane, it follows that if $f$ and $g$ are disjoint meromorphic functions in $U$, so are the functions $T f$ and $T_{g}$. Consequently, any necessary condition for disjointness satisfied by $f$ and $g$ has to be satisfied by $T f$ and $T g$. This will be the case, of course, if the necessary condition is expressed in terms of quantities which remain invariant under the transformation $f \rightarrow T f_{,} g \rightarrow T g$. Two quantities with this invariance property were given by Nehari [7], [8], namely,

Received by the editors November 2, 1972. $30 \mathrm{~A} 68$.

AMS (MOS) subject classifications (1970). Primary 30 A04, 34A20; Secondary 34C10,

(*) Posthumously. Professor Lavie died on December 14, 1973. 


$$
\phi(z)=\phi(f, g, z)=f^{\prime}(z) g^{\prime}(z) /[f(z)-g(z)]^{2},
$$

and

$$
\psi(z)=\psi(f, g, z)=\frac{f^{\prime \prime}(z)}{f^{\prime}(z)}-\frac{g^{\prime \prime}(z)}{g^{\prime}(z)}-2 \frac{f^{\prime}(z)+g^{\prime}(z)}{f(z)-g^{(z)}} .
$$

Another invariant quantity, which is not symmetric in $f$ and $g$, was given in [6], namely

$$
\theta(z)=\theta(f, g, z)=\frac{f^{\prime \prime}(z)}{f^{\prime}(z)}-\frac{2 f^{\prime}(z)}{f(z)-g(z)} .
$$

Moreover, it was shown in [6, Theorem 3], that if $\theta(z)=\theta(f, g, z)$ is regular, then every combination of $f(z)$ and $g(z)$ and their derivatives which remains invariant under the Möbius transformation $f \rightarrow T /, g \rightarrow T_{g}$, can be expressed in terms of $\phi(z)=\phi(f, g, z)$ and $\theta(z)=\theta(f, g, z)$ and their derivatives.

Turning to the problem of obtaining necessary conditions for disjoint functions we recall Nehari's results [8, Theorems 7.1 and 7.2]. If $f(z)$ and $g(z)$ are disjoint meromorphic functions in $U$, then

$$
|\phi(z)|=|\phi(f, g, z)| \leq 1 /\left(1-|z|^{2}\right)^{2}, \quad|z|<1 .
$$

The result is sharp, as can easily be checked for $f(z)=z$ and $g(z)=z^{-1}$. In order to obtain a bound for $\psi(z)$ one has to add further assumptions which will guarantee the regularity of $\psi(z)$ in $U$. Thus, assuming that $f(z)$ and $g(z)$ are univalent disjoint meromorphic functions in $U$, Nehari proved that

$$
|\psi(z)|=|\psi(f, g, z)|<8 /\left(1-|z|^{2}\right), \quad|z|<1 .
$$

The constant 8 is the best possible. In Theorem 4 we shall prove that if $f(z)$ and $g(z)$ are disjoint meromorphic functions (not necessarily univalent) in $U$, then

$$
|\phi(z) \psi(z)|=|\phi(f, g, z) \psi(f, g, z)| \leq 2 /\left(1-|z|^{2}\right)^{3}, \quad|z|<1 \text {, }
$$

and the result is sharp.

Following Nehari we shall first be interested in obtaining combinations of $f$ and $g$ and their derivatives which remain invariant under the transformation $f \rightarrow T /, g \rightarrow T g$. In $\$ 3$ we construct an infinite sequence of invariant quantities of this type which we call "invariant differential operators" and denote by $a_{n}(f, g, z), n=1,2, \ldots$. In order to obtain bounds for $a_{n}(f, g, z)$ we make use of pairs of functions.

Let

$$
P(z)=\sum_{k=1}^{\infty} a_{k} z^{k} \text { and } Q(z)=\sum_{k=1}^{\infty} b_{k} z^{k}, \quad|z|<1,
$$

be regular in the unit disk $U$, such that 


$$
P(z) Q(\zeta) \neq 1,|z|,|\zeta|<1,
$$

then we say that $(P, Q)$ is a pair [3]. We note that if $P=Q$, we have a BieberbachEilenberg function [2].

Pairs of functions and disjoint meromorphic functions are closely related. Indeed, if $f(z)$ and $g(z)$ are both regular at $z=0$, set

$$
P(z)=\frac{f(z)-f(0)}{f(z)-g(0)}, \quad Q(z)=\frac{g(z)-g(0)}{g(z)-f(0)}
$$

It is easily verified that $P(z)$ and $Q(z)$ defined in (2.10) form a pair if and only if $f(z)$ and $g(z)$ are disjoint meromorphic functions in $U$. We note that if the disjoint functions $f$ and $g$ are not both regular at $z=0$, then this situation may be achieved by means of a transformation $f \rightarrow T f, g \rightarrow T_{g}$.

We shall need the following theorem for pairs of functions. If $P(z)$ and $Q(z)$ are given by (2.8) and satisfy (2.9) then

$$
\sum_{k=1}^{\infty}\left|a_{k}\right|^{2} \sum_{k=1}^{\infty}\left|b_{k}\right|^{2} \leq 1 \text {. }
$$

For the proof of (2.11) in the case $P=Q$, see [2], [0]. In the more general case the proof is similar [11].

3. Invariant differential operators. In this section we construct a sequence of invariant differential operators $a_{n}(f, g, z), n=1,2, \ldots$. These operators are rational functions of the disjoint meromorphic functions $f$ and $g$ and their derivatives which remain invariant under the transformation $f \rightarrow T f, g \rightarrow T g$. Some properties of these invariant operators are considered and bounds are obtained. The ideas are similar to those in [1], where a similar technique was applied to a single univalent meromorphic function in $U$.

Theorem 1. Let

$$
R(f, g, w, z)=\frac{f^{\prime}(w)}{g(z+w)-f(w)}=\sum_{n=0}^{\infty} a_{n}(f, g, w) z^{n}, \quad|w|<1,|z+w|<1,
$$
where $f$ and $g$ are disjoint meromorpbic functions in $U$. Then $\alpha_{n}(f, g, w), n=$ $1,2, \cdots$, are invariant differential operators, which satisfy

$$
a_{n}^{\prime}=(n+1) a_{n+1}+\sum_{k=1}^{n-1} a_{k} a_{n-k}+a_{n} \theta, \quad n=1,2, \ldots,
$$

where $a_{n}=a_{n}(w)=a_{n}(f, g, w), \theta=\theta(\omega)=\theta(f, g, w)$ and

$$
a_{1}(f, g, w)=a_{1}(g, f, w)=-\phi(f, g, w) \text {. }
$$

Proof. Cf. [1, Theorem 1]. We assume first that $f$ and $g$ are both regular at the point $w$, and therefore the power series given in (3.1) converges in a neighborhood of $w$. Differentiation of (3.1) yields 


$$
\frac{\partial R}{\partial \omega}-\frac{\partial R}{\partial z}=\frac{f^{\prime \prime}(w) R}{f^{\prime}(w)}+R^{2}, \quad R=R(f, g, w, z) .
$$

Using (3.1), we substitute the power series expansion of each term in (3.4) and equate coefficients. This leads us to

$$
\begin{aligned}
a_{n}^{\prime}-(n+1) a_{n+1} & =\frac{f^{\prime \prime}(w) a_{n}}{f^{\prime}(w)}+\sum_{k=0}^{n} a_{k} a_{n-k} \\
& =\left[\frac{f^{\prime \prime}(w)}{f^{\prime}(w)}+2 a_{0}\right] a_{n}+\sum_{k=1}^{n-1} a_{k} a_{n-k}=\theta a_{n}+\sum_{k=1}^{n-1} a_{k} a_{n-k},
\end{aligned}
$$

and (3.2) is proved. (3.3) follows immediately from (3.1) and (2.3). The invariance of $a_{n}(f, g, w), n=1,2, \cdots$, follows from (3.2), (3.3) and the invariance of $\phi(f, g, w)$ and $\theta(f, g, w)$. We note that (3.2) and (3.3) hold also for a point $w$, $|w|<1$, such that $f^{\prime}(w)=0$. Indeed, in this case, we have $a_{n}(f, g, w)=0$, $n=0,1,2, \cdots$, while $\theta(f, g, w)$ has a simple pole. Hence the product $\alpha_{n}(w) \theta(w)$ as well as all the other terms of (3.2) are regular at an arbitrary point $w$, $|w|<1$. Finally, since $a_{n}(f, g, w), n=1,2, \cdots$, are invariant, (3.2) and (3.3) hold without the assumption of regularity of $f$ and $g$ at the point $w$.

Theorem 2. Let $f(z)$ and $g(z)$ be disjoint meromorpbic functions in $U$, which are regular at $z=0$. Let $P(z)=\sum_{n=1}^{\infty} a_{n} z^{n}$ and $Q(z)=\sum_{n=1}^{\infty} b_{n} z^{n}$ be defined by (2.10). If $a_{n}(f, g, w), n=1,2, \cdots$, is defined by (3.1), then

$$
a_{n}(0)=a_{n}(f, g, 0)=a_{1} b_{n}, \quad n=1,2, \ldots,
$$

and

Proof. By (3.1) we have

$$
\sum_{n=1}^{\infty}\left|a_{n}(f, g, 0)\right|^{2} \leq 1
$$

$$
R-a_{0}=\sum_{n=1}^{\infty} a_{n}(f, g, w) z^{n}=\frac{f^{\prime}(w)}{f(w)-g(w)} \cdot \frac{g(z+w)-g(w)}{g(z+w)-f(w)} .
$$

Hence,

$$
\sum_{n=1}^{\infty} a_{n}(f, g, 0) z^{n}=\frac{f^{\prime}(0)}{f(0)-g(0)} \cdot \frac{g(z)-g(0)}{g(z)-f(0)}=P^{\prime}(0) Q(z)=a_{1} \sum_{n=1}^{\infty} b_{n} z^{n},
$$

and (3.5) follows. Combining (3.5) and (2.11) we obtain (3.6)。

In the next theorem we consider the effect of a linear transformation of the independent variable $z$ on the coefficients $a_{n}(f, g, z)$.

Theorem 3. Let $f(z)$ and $g(z)$ be disjoint meromorphic functions in the unit disk $|z|<1$. Let

$$
t(z)=(z-\zeta) /(1-z \bar{\zeta}), \quad|z|<1,|\zeta|<1
$$


and set

$$
f(z)=F[t(z)], \quad g(z)=G[t(z)] .
$$

Then $F(t)$ and $G(t)$ are disjoint meromorpbic functions for $|t|<1$, and

$$
a_{n}\left(f, g_{l} z\right)=\sum_{k=1}^{n} \frac{\left(\begin{array}{c}
n-1 \\
k-1
\end{array}\right)\left(1-|\zeta|^{2}\right)^{k+1}(\zeta)^{n-k} a_{k}(F, G, t)}{(1-\bar{\zeta} z)^{n+k+2}}, n=1,2, \ldots
$$

Proof. Cf. [1, Theorem 2]. Let

$$
w_{0}=t\left(z_{0}\right)=\frac{z_{0}-\zeta}{1-\bar{\zeta} z_{0}}, \quad w+w_{0}=t\left(z+z_{0}\right)=\frac{z+z_{0}-\zeta}{1-\bar{\zeta}\left(z+z_{0}\right)}
$$

where $\left|z_{0}\right|<1$ and $z$ small enough such that $\left|z+z_{0}\right|<1$. It follows from (3.10) that

$$
w=z\left(1-|\zeta|^{2}\right) /\left[1-\bar{\zeta}\left(z+z_{0}\right)\right]\left(1-\bar{\zeta} z_{0}\right)
$$

According to (3.8) and (3.10), we have

$$
\begin{array}{ll}
f\left(z_{0}\right)=F\left(w_{0}\right), & f^{\prime}\left(z_{0}\right)=F^{\prime}\left(\dot{w}_{0}\right)\left(1-|\zeta|^{2}\right) /\left(1-\bar{\zeta} z_{0}\right)^{2}, \\
g\left(z_{0}\right)=G\left(w_{0}\right), & g\left(z+z_{0}\right)=G\left(w+w_{0}\right) .
\end{array}
$$

Hence,

$$
\frac{f^{\prime}\left(z_{0}\right)}{g\left(z+z_{0}\right)-f\left(z_{0}\right)}=\sum_{n=0}^{\infty} a_{n}\left(f, g, z_{0}\right) z^{n}=\frac{1-|\zeta|^{2}}{\left(1-\bar{\zeta} z_{0}\right)^{2}} \frac{F^{\prime}\left(w_{0}\right)}{G\left(w+w_{0}\right)-F\left(w_{0}\right)} .
$$

Since,

$$
\frac{F^{\prime}\left(w_{0}\right)}{G\left(w+w_{0}\right)-F\left(w_{0}\right)}=\sum_{n=0}^{\infty} a_{n}\left(F, G, w_{0}\right) w^{n}
$$

it follows from (3.11), (3.12) and (3.13) that

$$
\sum_{n=0}^{\infty} a_{n}\left(f, g, z_{0}\right) z^{n}=\frac{1-|\zeta|^{2}}{\left(1-\bar{\zeta} z_{0}\right)^{2}} \sum_{n=0}^{\infty} \frac{a_{n}\left(F, G, w_{0}\right)\left(1-|\zeta|^{2}\right)^{n} z^{n}}{\left(1-\bar{\zeta} z_{0}\right)^{n}\left[1-\bar{\zeta} z_{0}-\bar{\zeta} z\right]^{n}} .
$$

Using the power series expansion

$$
\frac{1}{(1-a)^{n}}=\sum_{k=0}^{\infty}\left(\begin{array}{c}
n+k-1 \\
k
\end{array}\right) a^{k}, \quad n=0,1,2, \ldots,
$$

for $a=\bar{\zeta} z /\left(1-\zeta z_{0}\right)$, and equating coefficients in (3.14) we obtain (3.9) for the arbitrary point $z=z_{0},\left|z_{0}\right|<1$ and its image $t=w_{0^{\circ}}$. For the details see [1, proof of Theorem 2].

Corollary. For $z_{0}=\zeta, w_{0}=0,(3.9)$ yields 


$$
a_{n}(f, g, \zeta)=\sum_{k=1}^{\infty} \frac{\left(\begin{array}{c}
n-1 \\
k-1
\end{array}\right)(\bar{\zeta})^{n-k} a_{k}(F, G, 0)}{\left(1-|\zeta|^{2}\right)^{n+1}}, n=1,2, \ldots
$$

Since by (3.6) $\left|a_{k}(F, G, 0)\right| \leq 1, k=1,2, \cdots$, it follows that

$$
\left|a_{n}(f, g, \zeta)\right| \leq(1+|\zeta|)^{n-1} /\left(1-|\zeta|^{2}\right)^{n+1}, n=1,2, \ldots,|\zeta|<1 .
$$

Moreover, if for a fixed $\zeta,|\zeta|<1$ we have $a_{k}(f, g, \zeta)=0, k=1, \cdots, n-1$ then, according to (3.9) ${ }^{\circ}, a_{k}(F, G, 0)=0, k=1,2, \cdots, n-1$, and

$$
\left|a_{n}(f, g, \zeta)\right|=\frac{\left|a_{n}(F, G, 0)\right|}{\left(1-|\zeta|^{2}\right)^{n+1}} \leq \frac{1}{\left(1-|\zeta|^{2}\right)^{n+1}} .
$$

4. Disjoint functions and nonoscillatory systems. In this section we apply the technique developed in $\$ 3$ to yield a necessary condition for two meromorphic functions to be disjoint in the unit disk (Theorem 4). Using this condition we then obtain necessary conditions for a differential system to be nonoscillatory (Theorem 5).

Theorem 4. Let $f(z)$ and $g(z)$ be disjoint meromorpbic functions in the unit disk. Let

$$
\begin{aligned}
\mu(f, g, z) & =\mu(z)=\phi(f, g, z) \psi(f, g, z) \\
& =\frac{f^{\prime \prime}(z) g^{\prime}(z)-g^{\prime \prime}(z) f^{\prime}(z)}{[f(z)-g(z)]^{2}}-\frac{2\left[f^{\prime}(z)+g^{\prime}(z)\right] f^{\prime}(z) g^{\prime}(z)}{[f(z)-g(z)]^{3}}
\end{aligned}
$$

Then

$$
|\mu(f, g, z)|=|\mu(z)| \leq 2 /\left(1-|z|^{2}\right)^{3}, \quad|z|<1,
$$

and the result is sharp.

Proof. Since $\mu(f, g, z)$ is invariant under the Möbius transformation $f \rightarrow T f$, $g \rightarrow T_{g}$, we may assume, without loss of generality, that $f(z)$ and $g(z)$ are regular at an arbitrary given point $\zeta,|\zeta|<1$. By (3.2) we have $a_{1}^{\prime}(f, g, \zeta)=$ $2 a_{2}(f, g, \zeta)+\alpha_{1}(f, g, \zeta) \theta(f, g, \zeta)$.

Interchanging the roles of $f(z)$ and $g(z)$ and using (3.3), we obtain

$$
\begin{aligned}
2\left[a_{2}(f, g, \zeta)-a_{2}(g, f, \zeta)\right] & =-\alpha_{1}(f, g, \zeta)[\theta(f, g, \zeta)-\theta(g, f, \zeta)] \\
& =\phi(f, g, \zeta) \psi(f, g, \zeta) .
\end{aligned}
$$

We first prove (4.3) for $z=0$. We assume that $f(z)$ and $g(z)$ are regular at $z=0$ and define $P(z)=\sum_{n=1}^{\infty} a_{n} z^{n}$ and $Q(z)=\sum_{n=1}^{\infty} b_{n} z^{n}$ as in (2.10). Since $f(z)$ and $g(z)$ are disjoint functions, $(P, Q)$ is a pair and by. Theorem 2 we have $a_{2}(f, g, 0)=a_{1} b_{2}, a_{2}(g, f, 0)=a_{2} b_{1}$. Hence,

$$
\begin{aligned}
\left|a_{2}(f, g, 0)-a_{2}(g, f, 0)\right| & =\left|a_{1} b_{2}-a_{2} b_{1}\right| \\
& \leq \sqrt{\left|a_{1}\right|^{2}+\left|a_{2}\right|^{2}} \sqrt{\left|b_{1}\right|^{2}+\left|b_{2}\right|^{2}} \leq 1,
\end{aligned}
$$


where (2.11) was used in the last step. (4.2), (4.4) and (4.5) imply $|\mu(0)| \leq 2$.

In order to prove (4.3) for an arbitrary point $\zeta,|\zeta|<1$, we apply the transformation (3.7). (3.9) and the identity $\alpha_{1}(F, G, 0)=a_{1}(G, F, 0)$ lead us to (4.6) $, a_{2}(f, g, \zeta)-a_{2}(g, f, \zeta)=\left(a_{2}(F, G, 0)-a_{2}(G, F, 0)\right) /\left(1-|\zeta|^{2}\right)^{3}, \quad|\xi|<1$.

Since by Theorem 3, $F(t)$ and $G(t)$ are disjoint meromorphic functions in the unit disk, we have according to (4.5)

$$
\left|a_{2}(F, G, 0)-\alpha_{2}(G, F, 0)\right| \leq 1
$$

(4.4), (4.6) and (4.5)' imply now (4.3) for an arbitrary point $\zeta,|\zeta|<1$.

It is easily checked that for $f(z)=z$ and $g(z)=z^{-2}$, we have $\mu(z)=$ $2\left(1+z^{3}\right) /\left(1-z^{3}\right)^{3}$, and $\mu(0)=2$. Moreover, if we set $f(z)=(z-\zeta) /(1-z \bar{\zeta})$, $g(z)=((z-\zeta) /(1-z \bar{\zeta}))^{-2},|\zeta|<1$, it follows from $(4.6)$ that $\mu(\zeta)=2\left(1-|\zeta|^{2}\right)^{-3}$. (This can also be verified by direct computation.) Hence, $(4.3)$ is sharp for an arbitrary $\zeta,|\zeta|<1$.

Remark. We note that (4.6) implies the transformation rule for the quantity $\mu(f, g, z)$; namely, if $F(t)$ and $G(t)$ are defined as in Theorem 3, then $\mu(f, g, z)\left(1-|z|^{2}\right)^{3}=\mu(F, G, t)\left(1-|t|^{2}\right)^{3}$. This, of course, follows also directly from the transformation formulas for $\phi(f, g, z)$ and $\psi(f, g, z)$ [8].

We apply now Theorem 4 in order to obtain necessary conditions for nonoscillation of the differential system

$$
w^{\prime}(z)=A(z) w(z)
$$

in the unit disk $U$. Here $w(z)$ is the column vector $\left[w_{1}(z), w_{2}(z), \cdots, w_{n}(z)\right]$ and $A(z)$ is the $n \times n$ matrix $\left[a_{i k}(z)\right]_{1}^{n}$, where $a_{i k}(z), i, k=1,2, \cdots, n$ are $n^{2}$ regular functions in the unit disk. (4.7) is called nonoscillatory in $U$, if for every choice of $n$ (not necessarily distinct) points $z_{1}, \cdots, z_{n}$ in $U$, the solution of (4.7) which satisfies $w_{i}\left(z_{i}\right)=0, i=1,2, \cdots, n$, is the trivial one $w(z) \equiv 0$. Nonoscillatory differential systems were called disconjugate in [10], [6].

In [6, Theorem 5], it was proved that if the system (4.7) is nonoscillatory in the unit disk $U$, then

$$
\left|a_{j k}(z) a_{k j}(z)\right| \leq 1 /\left(1-|z|^{2}\right)^{2}, \quad j \neq k, \quad j, k=1, \cdots, n, \quad|z|<1 .
$$

Using similar ideas we now prove:

Theorem 5. Let $a_{i k}(z), i, k=1, \cdots, n$, be regular functions in the unit disk $U$. If the system (4.7) is nonoscillatory in $U$, then 


$$
\begin{aligned}
\mid a_{k j}^{\prime}(z) a_{j k}(z) & -a_{j k}^{\prime}(z) a_{k j}(z)+2\left[a_{j j}(z)-a_{k k}(z)\right] a_{j k}(z) a_{k j}(z) \\
& +\sum_{i=1}^{n}\left[a_{k i}(z) a_{i j}(z) a_{j k}(z)-a_{j i}(z) a_{i k}(z) a_{k j}(z)\right] \mid \\
& \leq \frac{2}{\left(1-|z|^{2}\right)^{3}}, \quad|z|<1 .
\end{aligned}
$$

Proof. Cf. $\left[6\right.$, Theorems 4 and 5]. Let $u=\left[u_{1}, \cdots, u_{n}\right]$ and $v=\left[v_{1}, \cdots, v_{n}\right]$ be linearly independent solutions of (4.7) which satisfy at the point $\zeta \in U$ the following initial conditions.

$$
u_{i}(\zeta)=\delta_{i k}, \quad v_{i}(\zeta)=\delta_{i j}, \quad j \neq k, \quad i=1, \ldots, n,
$$

where $j$ and $k$ are fixed indices $j \neq k, j, k=1, \cdots, n$. Let

$$
f(z)=u_{j}(z) / v_{j}(z), \quad g(z)=u_{k}(z) / v_{k}(z) .
$$

Since the system (4.7) is nonoscillatory in $U$, it follows from [6, Theorem 2] that the meromorphic functions $f(z)$ and $g(z)$ defined in (4.11) are disjoint in $U$. This assertion can easily be confirmed. Indeed, suppose that $f\left(z_{j}\right)=g\left(z_{k}\right)=\alpha \beta^{-1}$ where $z_{j}, z_{k} \in U$, then the nontrivial solution $w(z)=\alpha u(z)-\beta v(z)$ satisfies $w_{i}(\zeta)=0, i \neq j, k, w_{j}\left(z_{j}\right)=w_{k}\left(z_{k}\right)=0$, which contradicts our assumption that the system (4.7) is nonoscillatory in $U$.

Using (4.7), (4.10) and (4.11) it follows [6, Theorem 4] that $\phi(f, g, \zeta)=$ $-a_{j k}(\zeta) a_{k j}(\zeta)$, and

$$
\theta(f, g, \zeta)=\frac{a_{j k}^{\prime}(\zeta)}{a_{j k}(\zeta)}+\frac{\sum_{i=1}^{n} a_{j i}(\zeta) a_{i k}(\zeta)}{a_{j k}(\zeta)}-2 a_{j j}(\zeta)
$$

Hence

$$
\begin{aligned}
\mu(f, g, \zeta)= & {[\theta(f, g, \zeta)-\theta(g, f, \zeta)] \phi(f, g, \zeta) } \\
= & a_{k j}^{\prime}(\zeta) a_{j k}(\zeta)-a_{j k}^{\prime}(\zeta) a_{k j}(\zeta) \\
& +\sum_{i=1}^{n}\left[a_{k i}(\zeta) a_{i j}(\zeta) a_{j k}(\zeta)-a_{j i}(\zeta) a_{i k}(\zeta) a_{k j}(\zeta)\right] \\
& +2\left[a_{j j}(\zeta)-a_{k k}(\zeta)\right] a_{j k}(\zeta) a_{k j}(\zeta) .
\end{aligned}
$$

The result follows now from (4.12) and (4.3).

To see that (4.9) is sharp we consider the following differential system

$$
w^{\prime}(z)=\left(\begin{array}{lc}
0 & a_{12}(z) \\
a_{21}(z) & 0
\end{array}\right) w(z)
$$


In this case $(4.9)$ reduces to

$$
\left|a_{12}^{\prime}(z) a_{21}(z)-a_{21}^{\prime}(z) a_{12}(z)\right| \leq 2 /\left(1-|z|^{2}\right)^{3}, \quad|z|<1 .
$$

It is easily checked that if

$$
a_{12}(z)=\frac{1}{\left(1-z^{m+2}\right)^{2 /(m+2)}}, \quad a_{21}(z)=\frac{(m+1) z^{m}}{\left(1-z^{m+2}\right)^{2-2 /(m+2)}},
$$

$$
m=0,1, \ldots,
$$

then the general solution of (4.7) is given by

$$
\begin{aligned}
& w_{1}(z)=\left(1-z^{m+2}\right)^{-1 /(m+2)}\left(c_{1} z+c_{2}\right), \\
& w_{2}(z)=\left(1-z^{m+2}\right)^{-1-1 /(m+2)}\left(c_{1}+c_{2} z^{m+1}\right) .
\end{aligned}
$$

Evidently, $w_{1}(z)$ and $w_{2}(z)$ cannot both vanish at the unit disk unless $c_{1}=c_{2}=0$, and therefore the differential system (4.7) ${ }^{\prime}$ is nonoscillatory for any choice of integer $m=0,1,2, \cdots$ in (4.13). Substituting (4.13) in (4.9)', we find that equality holds for $m=1$ and $z=0$. Hence (4.9) ${ }^{\prime}$ is sharp. Sharpness of (4.9) for $n \times n$ matrices $A(z)$ will be discussed in the next section.

5. Disfocal differential equations. In the particular case where

$$
A(z)=\left[\begin{array}{cccccccc}
0 & 1 & 0 & \cdot & \cdot & \cdot & \cdot & 0 \\
0 & 0 & 1 & \cdot & \cdot & \cdot & \cdot & 0 \\
\cdot & \cdot & \cdot & \cdot & \cdot & \cdot & \cdot & \cdot \\
0 & 0 & \cdot & \cdot & \cdot & \cdot & 1 & 0 \\
0 & 0 & 0 & \cdot & \cdot & \cdot & 0 & 1 \\
-q_{n} & -q_{n-1} & \cdot & \cdot & \cdot & \cdot & -q_{2} & -q_{1}
\end{array}\right]
$$

the column vector $w(z)=\left[w_{1}(z), \cdots, w_{n}(z)\right]$ becomes $\left[y(z), y^{\prime}(z), \cdots, y^{(n-1)}(z)\right]$ and (4.7) is equivalent to the differential equation

$$
y^{(n)}(z)+q_{1}(z) y^{(n-1)}(z)+\cdots+q_{n}(z) y(z)=0 .
$$

In this case nonoscillation of (4.7) in $U$, is equivalent to disfocality of (5.2) there. In the following we assume that the coefficients $q_{k}(z), k=1, \cdots, n$, are regular functions in the unit disk $U$. (5.2) is called disfocal in $U$, if for every choice of $n$ (not necessarily distinct) points $z_{1}, \ldots, z_{n}$ of $U$, the only solution of (5.2) which satisfies $y\left(z_{1}\right)=y^{\prime}\left(z_{2}\right)=\cdots=y^{(n-1)}\left(z_{n}\right)^{n}=0$, is the trivial one $y(z) \equiv 0$. Necessary conditions for disfocality of equation $(5.2)$ in $U$ were obtained in [6, Theorem 7].

We apply now Theorem 5 to the differential equation (5.2). We first set $j=n-1, k=n$ in (4.9) and use (5.1). This yields the following result which we state as 
Theorem 5'. If the differential equation (5.2) is disfocal in the unit disk $U$, then

$$
\left|2 q_{1}(z) q_{2}(z)+q_{2}^{\prime}(z)+q_{3}(z)\right| \leq 2 /\left(1-|z|^{2}\right)^{3}, \quad|z|<1 .
$$

If $n=2$, (5.3) reduces to

$$
\left|2 q_{1}(z) q_{2}(z)+q_{2}^{\prime}(z)\right| \leq 2 /\left(1-|z|^{2}\right)^{3}, \quad|z|<1 .
$$

(5.3) is sharp for a differential equation of any order $n \geq 2$, as will be seen later.

We next set $j=n-2, k=n$ in (4.9). According to (5.1) this yields another necessary condition for disfocality of (5.2) in $U$; namely $\left|q_{3}(z)\right| \leq 2 /\left(1-|z|^{2}\right)^{3}$, $|z|<1$. We shall show now that this condition can also be obtained in a slightly different way and we prove the following more general result.

Theorem 6. If equation (5.2) is disfocal in the unit disk $U$, then

$$
\left|q_{k}(z)\right| \leq(k-1) ! /\left(1-|z|^{2}\right)^{k}, \quad|z|<1, k=2, \cdots, n .
$$

Proof. Let $u(z)$ and $v(z)$ be linearly independent solutions of (5.2), which satisfy at the point $\zeta \in U$ the following initial conditions

$$
u^{(i)}(\zeta)=\delta_{i j}, \quad v^{(i)}(\zeta)=\delta_{i, j-1}, \quad i=0, \ldots, n-1,
$$

where $j$ is a fixed index such that $1 \leq j \leq n-1$. Let

$$
f(z)=u^{(j-1)}(z) / v^{(j-1)}(z), \quad g(z)=u^{(j)}(z) / v^{(j)}(z) .
$$

Since equation (5.2) is disfocal in $U$, it follows from [6, Theorem 2], that the meromorphic functions $f(z)$ and $g(z)$ defined in (5.7) are disjoint in.U. Moreover, according to (5.6) and (5.7) $f(\zeta)=0, f^{\prime}(\zeta)=1$ and therefore

$$
\begin{aligned}
\sum_{n=0}^{\infty} \alpha_{n}(f, g, \zeta) z^{n} & =\frac{f^{\prime}(\zeta)}{g(z+\zeta)-f(\zeta)}=\frac{1}{g(z+\zeta)}=\frac{v^{(j)}(z+\zeta)}{u^{(j)}(z+\zeta)} \\
& =\frac{v^{(j)}(\zeta)+v^{(j+1)}(\zeta) z+\cdots}{u^{(j)}(\zeta)+u^{(j+1)}(\zeta) z+\cdots}
\end{aligned}
$$

Using the initial conditions (5.6) and the differential equation (5.2), it follows that Hence,

$$
\sum_{n=0}^{\infty} a_{n}(f, g, \zeta) z^{n}=\frac{v^{(n)}(\zeta) z^{n-j} /(n-j) !+\cdots}{1+u^{(j+1)}(\zeta) z+\cdots}=\frac{-q_{n-j+1}(\zeta) z^{n-j}}{(n-j) !}+\cdots
$$

$$
a_{k}(f, g, \zeta)=0, \quad k=1, \ldots, n-j-1, \quad a_{n-j}(f, g, \zeta)=-q_{n-j+1}(\zeta) /(n-j) !
$$

Applying now the corollary of Theorem 3 , it follows from (3.16) that 


$$
\left|\alpha_{n-j}(f, g, \zeta)\right|=\left|q_{n-j+1}(\zeta) /(n-j) !\right| \leq 1 /\left(1-|\zeta|^{2}\right)^{n-j+1}
$$

Since $\zeta$ is an arbitrary point of the unit disk $U$, and $j$ is an arbitrary index such that $1 \leq j \leq n-1,(5.8)$ completes the proof of Theorem 6 .

For $k=2,(5.5)$ is known [6], and for $k=3, \cdots, n$, Theorem 6 improves the result obtained in [6, Theorem 7]. The technique applied there was slightly different; namely, it was shown [6, Theorem 6] that if $f(z)$ and $g(z)$ are defined as in (5.7) and $\phi(z)=\phi(f, g, z)$, then

$$
\phi(\zeta)=\phi^{\prime}(\zeta)=\cdots=\phi^{(n-j-2)}(\zeta)=0
$$

and

$$
\phi^{(n-j-1)}(\zeta)=q_{n-j+1}(\zeta)
$$

In view of (5.9) and (5.10), Theorem 6 is an immediate corollary of the following result.

Theorem 6'. Let $f(z)$ and $g(z)$ be disjoint meromorpbic functions in the unit disk. Assume that $\theta(z)=\theta(f, g, z)$ is a regular function at the point $\zeta,|\zeta|<1$. If

$$
\phi(\zeta)=\phi^{\prime}(\zeta)=\cdots=\phi^{(m-1)}(\zeta)=0
$$

then

$$
\left|\phi^{(m)}(\zeta)\right| \leq(m+1) ! /\left(1-|\zeta|^{2}\right)^{m+2}
$$

(5.12) is sharp for an arbitrary point $\zeta,|\zeta|<1$ and any integer $m=0,1,2, \ldots$

Proof. Without loss of generality we may assume that $f(z)$ and $g(z)$ are both regular at the point $\zeta$. Hence, $\theta(f, g, z)$ is regular at the point $\zeta$ if and only if

$$
f^{\prime}(\zeta) \neq 0 \text {. }
$$

By the definition of $\phi(f, g, z)$, it follows now from (5.11) that $g^{\prime}(\zeta)=$ $g^{\prime \prime}(\zeta)=\cdots=g^{(m)}(\zeta)=0$. Therefore,

$\sum_{n=0}^{\infty} \alpha_{n}(f, g, \zeta) z^{n}=\frac{f^{\prime}(\zeta)}{g(z+\zeta)-f(\zeta)}=\frac{f^{\prime}(\zeta)}{g(\zeta)-f(\zeta)}\left[1-\frac{g^{(m+1)}(\zeta) z^{m+1}}{(m+1) !(g(\zeta)-f(\zeta))}+\cdots\right]$.

Hence, $\alpha_{k}(f, g, \zeta)=0, k=1,2, \cdots, m$, and

$$
\alpha_{m+1}(f, g, \zeta)=\frac{f^{\prime}(\zeta) g^{(m+1)}(\zeta)}{[g(\zeta)-f(\zeta)]^{2}(m+1) !}=-\frac{\phi^{(m)}(\zeta)}{(m+1) !}
$$

(5.12) follows now by the corollary of Theorem 3.

To show the sharpness of (5.12) we consider the functions 


$$
f(z)=z, \quad g(z)=z^{-m-1}, \quad m=0,1,2, \ldots,
$$

which are disjoint in the unit disk. In this case, $\phi(f, g, z)=\phi(z)=-$ $(m+1) z^{m} /\left(1-z^{m+2}\right)^{2}$ has a zero of order $m$ at the origin and $\phi^{(m)}(0)=-(m+1)$ ! Moreover, for

$$
f(z)=\frac{z-\zeta}{1-z \bar{\zeta}}, \quad g(z)=\left(\frac{z-\zeta}{1-z \bar{\zeta}}\right)^{-m-1}, \quad|\zeta|<1,
$$

we find that $\phi(f, g, z)$ has a zero of order $m$ at the point $z=\zeta$ and $\phi^{(m)}(\zeta)=$ $-(m+1) ! /\left(1-|\zeta|^{2}\right)^{m+2}$.

Remarks. (i) Since $\phi(f, g, z)$ is symmetric in $f$ and $g$, we may interchange the roles of $f$ and $g$ and assume that $\theta(g, f, z)$ is regular at the point $\zeta$. Nevertheless, Theorem $\sigma^{\prime}$ does not hold if both $\theta(f, g, z)$ and $\theta(g, f, z)$ have poles at the point $z=\zeta$. Indeed, let $f(z)=2+z^{k}, g(z)=z^{k}, k=2,3, \ldots$ At the origin, both $\theta(f, g, z)$ and $\theta(g, f, z)$ have a simple pole, while $\phi(f, g, z)$ has a zero of order $(2 k-2)$ and $\phi^{(2 k-2)}(0)=k^{2}(2 k-2) ! / 4>(2 k-1) !$ for $k \geq 8$.

(ii) If we choose $f(z)$ and $g(z)$ as in $(5.7), f^{\prime}(\zeta)=1$, and Theorem 6 follows from Theorem $6^{\prime}$. We have seen that Theorem $6^{\prime}$ is sharp for an arbitrary integer $m$, yet all we can say with regard to the sharpness of Theorem 6 is that $(5.5)$ is sharp for $k=2$. This will be shown by the following example.

Consider the second order differential equation

$$
y^{\prime \prime}(z)+q_{1}(z) y^{\prime}(z)+q_{2}(z) y(z)=0 .
$$

Let $u(z)$ and $v(z)$ be linearly independent solutions of (5.14) and set

$$
f(z)=u(z) / v(z), \quad g(z)=u^{\prime}(z) / v^{\prime}(z) .
$$

It is easily verified [6, Theorem 6$]$ that

$$
q_{2}(z)=\phi(f, g, z), \quad q_{1}(z)=-\theta(f, g, z),
$$

and equation (5.14) is disfocal in $U$ if and only if $f(z)$ and $g(z)$ are disjoint meromorphic functions in $U$ [8]. Hence, if we set $f(z)$ and $g(z)$ as in (5.13) it follows from $(5.16)$ that the differential equation

$$
y^{\prime \prime}(z)-\frac{2 z^{m+1}}{1-z^{m+2}} y^{\prime}(z)-\frac{(m+1) z^{m}}{\left(1-z^{m+2}\right)^{2}} y(z)=0, \quad m=0,1, \ldots,
$$

is disfocal in $U$. For $m=0,(5.17)$ yields $q_{2}(z)=-\left(1-z^{2}\right)^{-2}$, which shows that the bound for $\left|q_{2}(z)\right|$ given in (5.5) is sharp for second order differential equations.

Combining Theorem $6^{\prime}$ and (5.16) we obtain the following corollary. If equation (5.14) is disfocal in the unit disk $U$ and if at the point $\zeta \in U$, $(5.11)^{\prime} q_{2}(\zeta)=q_{2}^{\prime}(\zeta)=\cdots=q_{2}^{(m-1)}(\zeta)=0, \quad q^{(m)}(\zeta) \neq 0, \quad m=0,1,2, \cdots$ 
then

$$
\left|q_{2}^{(m)}(\zeta)\right| \leq(m+1) ! /\left(1-|\zeta|^{2}\right)^{m+2} .
$$

Cf. [5, Theorem 5']. Equation (5.17) shows that (5.12) is sharp for any integer $m$. We note that the bounds, for the coefficients of a disfocal equation given in Theorems $5^{\prime}$ and 6 do not depend on the order of the differential equation. In view of this, the following observation may be of interest. The differential equations

$$
\eta^{(s)}(z)+q_{1}(z) \eta^{(s-1)}(z)+\cdots+q_{s}(z) \eta(z)=0, \quad s \geq 2,
$$

and

$$
y^{(n)}(z)+q_{1}(z) y^{(n-1)}(z)+\cdots+q_{s}(z) y^{(n-s)}(z)=0, \quad n \geq s,
$$

are simultaneously disfocal in a given simply connected domain $D$. Indeed, if $y(z)$ satisfies (5.19) then $y^{(n-s)}(z)$ satisfies (5.18). Since $y^{(n-s)}(z)=0$ is disfocal in the whole complex plane, it follows that the differential equation $y^{(n-s)}(z)=\eta(z)$ has a unique solution, which satisfies $y\left(z_{1}\right)=y^{\prime}\left(z_{2}\right)=\cdots=$ $y^{(n-s)}\left(z_{n-s}\right)=0$ where $z_{1}, \cdots, z_{n-s}$ are arbitrary points of $D$. Consequently, the differential equations

$(5.17)^{\prime} y^{(n)}(z)-\frac{2 z^{m+1}}{1-z^{m+2}} y^{(n-1)}(z)-\frac{(m+1) z^{m}}{\left(1-z^{m+2}\right)^{2}} y^{(n-2)}(z)=0, \quad m=0,1, \ldots$, and (5.17) are simultaneously disfocal in $U$.

We use now equation (5.17) ${ }^{\prime}$ in order to discuss the sharpness of our results. First, we set $m=0$ in (5.17) $)^{\prime}$. It follows that (5.5) is sharp for $k=2$ and any order $n \geq 2$. Next, we set $m=1$. Since $q_{3}(z)=0$ in $(5.17)^{\prime}$, we now obtain that (5.3) is sharp. Finally, we reduce equation $(5.17)^{\prime}$ to a differential system of the form (4.7). The companion $n \times n$ matrix $A(z)$ is given by

$$
\begin{gathered}
A(z)=\left[\begin{array}{ccccccc}
0 & 1 & \cdot & \cdot & \cdot & 0 & 0 \\
0 & 0 & 1 & \cdot & \cdot & 0 & 0 \\
\cdot & \cdot & \cdot & \cdot & \cdot & \cdot & \cdot \\
0 & \cdot & \cdot & \cdot & \cdot & 0 & 1 \\
0 & \cdot & \cdot & \cdot & 0 & -q_{2} & -q_{1}
\end{array}\right], \\
q_{1}=\frac{-2 z^{m+1}}{1-z^{m+2}}, \quad q_{2}=\frac{-(m+1) z^{m}}{\left(1-z^{m+2}\right)^{2}}
\end{gathered}
$$

and the corresponding differential system (4.7) is nonoscillatory in the unit disk $U$. Setting $m=0$ and $m=1$ in (5.20) we conclude that (4.8) and (4.9) are both sharp 
for $j=n-1, k=n$. We note that the example given in the end of $\$ 4$ can be obtained from the example (5.20) by means of the transformation $\tilde{w}_{1}(z)=w_{1}(z)$, $\tilde{w}_{2}(z)=\left(1-z^{m+2}\right)^{2 /(m+2)} w_{2}(z)$, cf. [10, p. 489].

We conclude with the following example which shows that Theorems $5^{\prime}$ and 6 are independent. Consider the differential equation .

$$
y^{\prime \prime}(z)+\left((m+1) z^{m} /\left(1-z^{m+2}\right)^{2}\right) y(z)=0, \quad m=0,1, \ldots
$$

It is easily verified, cf. [4], that

$$
\left|(m+1) z^{m} /\left(1-z^{m+2}\right)^{2}\right| \leq 1 /\left(1-|z|^{2}\right)^{2}, \quad|z|<1, \quad m=0,1, \ldots,
$$

and therefore (5.21) satisfies the necessary condition for disfocality (5.5). Moreover, $q_{2}(z)$, the coefficient of $y(z)$ in (5.21) has a zero of order $m$. at the origin and condition (5.12)' is also satisfied. Yet, an elementary computation shows that if $0 \leq m<5$, then for real positive values $z=x, 0<x<1$, we have $\lim _{x \rightarrow 1} q_{2}^{\prime}(x)\left(1-x^{2}\right)^{3}>2$. Hence condition $(5.3)^{\prime}$ is not satisfied and therefore equation (5.21), where $m=0,1,2,3,4$, is not disfocal in the unit disk.

\section{REFERENCES}

1. D. Aharonov, A necessary and sufficient condition for univalence of a meromorphic function, Duke Math. J. 36 (1969), 599-604. MR 40 \#2865.

2. - On Bieberbach-Eilenberg functions, Bull. Amer. Math. Soc. 76 (1970), 101-104. MR $41 \# 1994$.

3. - A gen eralization of a theorem of J. A. Jenkins, Math. Z. 110 (1969), 218222. MR $40 \# 325$.

4. S. Friedland and Z. Nehari, Univalence conditions and Sturm-Liouville eigenvalues, Proc. Amer. Math. Soc. 24 (1970), 595-603. MR 40 \#4435.

5. M. Lavie, The Schwarzian derivative and disconjugacy of $n$-th order linear differential equations, Canad. J. Math. 21 (1969), 235-249. MR 38 \#6153.

6. - Some function theoretic aspects of disconjugacy of linear differential systems, Trans. Amer. Math. Soc. 143 (1969), 153-171. MR 40 \#2944.

7. Z. Nehari, Some inequalities in the theory of functions. Trans. Amer. Math. Soc. 75 (1953), 256-286. MR 15, 115.

8. - Some function-theoretic aspects of linear second order differential equations, J. Analyse Math. 18 (1967), 259-276. MR 35 \#4391.

9. - On the coefficients of Bieberbach-Eilenberg functions, J. Analyse Math. (1970), 297-303.

10. B. Schwarz, Disconjugacy of complex differential systems, Trans. Amer. Math. Soc. 125 (1966), 482-496. MR 34 \#6190.

11. J. Szargel, M. Sc. Thesi s, Technion, Israel Institute of Technology, 1970.

DEPARTMENT OF MATHEMATICS, TECHNION-ISRAEL INSTITUTE OF TECHNOLOGY, HAIFA, ISRAEL 